Mercedes Elizabeth Cárdenas-Pañi; Cecilia Ivonne Narváez-Zurita; Juan Carlos Erazo-Álvarez; Mireya Magdalena Torres-Palacios

http://dx.doi.org/10.35381/r.k.v5i10.715

\title{
Conciliación de impuestos a las ganancias. Un estudio bajo la normativa fiscal y normativa contable
}

\section{Reconciliation of income taxes. A study under tax regulations and accounting regulations}

Mercedes Elizabeth Cárdenas-Pañi mercedes.cardenas@psg.ucacue.edu.ec Universidad Católica de Cuenca, Cuenca

Ecuador

https://orcid.org/0000-0001-9540-4267

Cecilia Ivonne Narváez-Zurita inarvaez@ucacue.edu.ec

Universidad Católica de Cuenca, Cuenca

Ecuador

https://orcid.org/0000-0002-7437-9880

Juan Carlos Erazo-Álvarez

jcerazo@ucacue.edu.ec

Universidad Católica de Cuenca, Cuenca

Ecuador

https://orcid.org/0000-0001-6480-2270

Mireya Magdalena Torres-Palacios

mireya.torres@ucacue.edu.ec

Universidad Católica de Cuenca, Cuenca

Ecuador

https://orcid.org/0000-0002-4929-6960

Recibido: 23 de marzo de 2020

Revisado: 12 de abril de 2020

Aprobado: 05 de mayo de 2020

Publicado: 19 de mayo de 2020

\section{RESUMEN}

En Ecuador la aplicación de las Normas Internacionales de Información Financiera (NIIF) surge debido a la globalización de la economía. Este proceso ha generado algunas divergencias entre materia tributaria y contable, naciendo así los impuestos diferidos. En consecuencia, la presente investigación propone diseñar una guía para la 
conciliación del impuesto a las ganancias; así como el tratamiento contable y tributario de los impuestos diferidos desde su origen hasta la liquidación o recuperación en la empresa APC Tecnología, como mecanismo para ajustar las diferencias temporarias. Este estudio tuvo un enfoque cuantitativo con un alcance descriptivo, los instrumentos que se utilizaron fueron la encuesta y la revisión documental mediante el método analítico a los estados financieros. Entre los principales resultados se determinó que la empresa en estudio no ha realizado una aplicación integra de las NIIF para PYMES.

Descriptores: Tributación; administración de empresas; empresa privada; ganancia. (Palabras tomadas del Tesauro UNESCO).

\section{ABSTRACT}

In Ecuador, the application of International Financial Reporting Standards (IFRS) arises from the globalization of the economy. This process has generated some differences between tax and accounting matters, thus creating deferred taxes. In consequence, this investigation proposes to design a guide for the reconciliation of income tax, as well as the accounting and tax treatment of deferred taxes, from inception to the liquidation or recovery as a mechanism to adjust temporary differences in the APC Technology company. This study had a quantitative approach with a descriptive scope, the instruments were based on the survey and the documentary review, using the analytical method of the financial statements. Among the main results, it was determined that the company under study has not made a full application of the IFRS for SMEs.

Descriptores: Taxation; business management; private enterprises; profits. (Words taken from the UNESCO Thesaurus).

\section{INTRODUCCIÓN}

A lo largo de los años las ciencias contables han influenciado en las actividades económicas globales, gestando una historia que ha llevado a la creación colaborativa de normas internacionales aplicadas con ciertas diferencias a grandes potencias y a países en vías de desarrollo. Por su parte, las Normas Internacionales de Información Financiera (NIIF), nacidas en el año 2001, tienen como objetivo transparentar la información contable de las empresas (Chávez-Pullas \& Herrera-Estrella, 2018).

En el Ecuador, las NIIF se instauraron en el año 2008, según resolución de la Superintendencia de Compañías, Valores y Seguros (SUPERCIAS) 08.G.DSC.010 de 2008.11.20, R.O. No. 498. Su establecimiento obligó a la modificación en la 


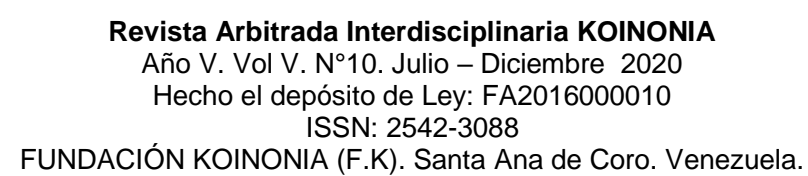

Mercedes Elizabeth Cárdenas-Pañi; Cecilia Ivonne Narváez-Zurita; Juan Carlos Erazo-Álvarez; Mireya Magdalena Torres-Palacios

presentación de la información financiera ya que su implantación promovió la inversión extranjera y posibilitó a las empresas nacionales ubicarse a la altura de sus pares internacionales.

Las empresas ecuatorianas empezaron el camino de transición de las Normas Ecuatorianas de Contabilidad (NEC) a las NIIF, adaptando sus cuentas contables y capacitando al personal para dar cumplimiento a las disposiciones del ente de control (SUPERCIAS), emitiendo información financiera de conformidad con la normativa contable vigente. De esta transición surgen divergencias entre la normativa contable y tributaria. El mayor inconveniente encontrado se origina al momento de liquidar el impuesto a las ganancias, es decir, al realizar el impuesto a la renta anual.

Dado lo anterior, el objetivo del presente artículo consiste en diseñar una guía contable para la conciliación tributaria del impuesto a las ganancias en la empresa APC Tecnología, como mecanismo de ajuste de las diferencias temporarias.

APC Tecnología es una compañía familiar constituida en el año 2007 en la ciudad de Cuenca - Ecuador, dedicada a la venta al por mayor y menor de equipos de computación, partes, piezas, módulos periféricos y mantenimiento de equipos tecnológicos. Su crecimiento hasta la actualidad ha sido considerable, ya que cuenta con tres locales comerciales en la ciudad de Cuenca y uno en la ciudad de Machala, por lo que resulta necesario regularizar las diferencias temporarias.

\section{Referencial teórico}

En la década de los cincuenta el crecimiento económico acelerado sumado a la globalización de la economía mundial, generó la necesidad de establecer un estándar que transparente la información contable de las empresas. En el año 1973 se reunió el International Accounting Standard Committee (IASC) con representantes de las principales economías de ese entonces. De esta reunión nacieron las Normas Internacionales de Contabilidad (NIC) con el objetivo de ser la base de las prácticas contables a nivel global para que las economías de todo el mundo, incluidos países en 
Mercedes Elizabeth Cárdenas-Pañi; Cecilia Ivonne Narváez-Zurita; Juan Carlos Erazo-Álvarez; Mireya Magdalena Torres-Palacios

desarrollo, generen información contable de calidad, tal como promueven (VílchezOlivares, 2007) y Ugalde-Binda, 2014).

Las intenciones del IASC fueron un éxito logrando que grandes empresas y corporaciones entregaran sus informes anuales basados en las 28 normas publicadas por la entidad hasta el año 1987 (Zeff, 2012). Sin embargo, más adelante se presentaron problemas dado que algunas empresas no se sentían representadas por la IASC o se consideraba que la normas expedidas no brindaban estándares reales, pues los diferentes tratamientos aplicados a una misma transacción generaban múltiples resultados (Richardson \& Eberlein, 2011), (Cabrera-Álvarez, Narváez-Zurita \& ErazoÁlvarez, 2020). En el año 2000 más de 140 profesionales contables de alrededor de 100 países a nivel mundial formaban la IASB (Accounting Standards Board) con el siguiente objetivo:

Desarrollar un único conjunto de normas contables de carácter global que sean de alta calidad, comprensibles y de cumplimiento obligado, que requieran información de alta calidad, transparente y comparable en los estados financieros y en otros tipos de información financiera para ayudar a los participantes en los mercados de capitales de todo el mundo y a otros usuarios a tomar decisiones económicas. (Organización Interamericana de Ciencias Económicas (OICE), 2020, p.1)

La IASB adoptó las NIC encargándose de mejorarlas y reemplazarlas por las NIIF, publicando hasta la presente fecha 13 nuevas normativas aplicadas tanto a grandes economías del mundo como a países en vías de desarrollo; esto se dio con el apoyo de comisiones de valores y la comunidad económica europea (García-Carvajal \& DueñasCasallas, 2016).

En el Ecuador, en agosto del año 2006 mediante resolución emitida por la SUPERCIAS., se establece la adopción de las NIIF con carácter obligatorio y se fija un cronograma de aplicación en tres grupos divididos por tipología de empresa que inician en el año 2010, hasta 2012, como se puede observar en la figura 1 (Campoverde-León, \& Castro-Garcés, 2016). 


\begin{tabular}{|rl|}
\hline Año & \multicolumn{1}{c|}{ Compañías } \\
\hline 2010 & $\begin{array}{l}\text { Sujetas por la ley de mercados y valores. } \\
\text { Ejercen actividad de auditoria externa. }\end{array}$ \\
\hline 2011 & $\begin{array}{l}\text { Con activos totales o iguales a } \$ 4.000 .000,00 \\
\text { al } 31 / 12 / 2007,\end{array}$ \\
\hline 2012 & $\begin{array}{l}\text { Lolding tenedoras de acciones. } \\
\text { anteriores. }\end{array}$ \\
\hline
\end{tabular}

Figura 1. Implementación NIIF en el Ecuador.

Fuente: Superintendencia de Compañias del Ecuador (2008).

Se encuentran exentas de la aplicación de las NIIF las sociedades civiles, personas naturales, entidades financieras y públicas (SUPERCIAS, 2010). En el año 2012 las NIIF se establecieron como las normas contables aceptadas en el Ecuador, su aplicación está ligada a la Ley Orgánica de Régimen Tributario Interno (LORTI), siendo el Servicio de Rentas Internas (SRI) su ente rector (Dirección Nacional Jurídica (DNJ), 2016).

Bajo el marco de estos antecedentes, en el Ecuador al realizar la conciliación entre la normativa contable NIC 12 (impuesto a las ganancias) y la normativa tributaria LORTI, su aplicación genera diferencias importantes, ya que persiguen distintos objetivos; las NIIF buscan mostrar fielmente la situación financiera de las empresas; mientras que, la normativa tributaria busca establecer la base imponible del impuesto a la renta sobre la cual se tributa y se liquidan impuestos (Mena, Narváez \& Erazo, 2019), (CabreraÁlvarez, Narváez-Zurita \& Erazo-Álvarez, 2020). .Al respecto, (Cóndor-Paucar, 2015) afirma que estas contraposiciones dan lugar a la aparición de los impuestos diferidos, por lo que es necesario resaltar la obligatoriedad de la aplicación conjunta de la norma contable y la normativa tributaria ecuatoriana. 
Mercedes Elizabeth Cárdenas-Pañi; Cecilia Ivonne Narváez-Zurita; Juan Carlos Erazo-Álvarez; Mireya Magdalena Torres-Palacios

\section{El impuesto a las ganancias según la normativa tributaria}

En la legislación ecuatoriana el periodo contable va desde el 1 de enero al 31 de diciembre, al finalizar éste, las empresas deben declarar los resultados obtenidos ante el SRI; mediante la conciliación tributaria se determina la base imponible para la liquidación del impuesto, es aquí, donde nacen las diferencias entre el marco contable y el marco tributario, por lo que es necesario identificar el tratamiento adecuado que debe aplicarse, puesto que, cualquier error afectaría el impuesto a pagar.

En este contexto, resulta imperioso entender cuál es la diferencia entre la normativa contable que se basa en NIC 12, NIIF para PYMES sección 29 y la normativa tributaria sustentada en la LORTI y sus reglamentos. El impuesto a las ganancias está relacionado con los lucros o pérdidas que se obtienen en el ejercicio fiscal. Tanto ganancias como pérdidas están sujetas a la tasa impositiva reglamentada en la normativa tributaria o impuesto a la renta. Esta grava todos los ingresos obtenidos, ya sea en el país o en el exterior, por las personas naturales y jurídicas establecidas en el Ecuador, tanto por las actividades económicas que desempeñen como por los ingresos que hayan obtenido a título gratuito u oneroso. La LORTI establece los ingresos gravados y exentos (ver figura 2) para la liquidación de este impuesto, dado que, los ingresos gravados son la base para el cálculo del impuesto a la renta.

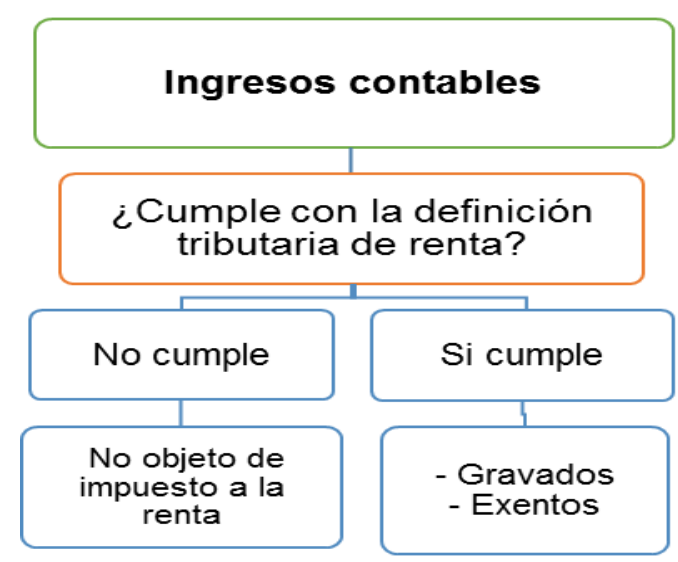

Figura 2. Reconocimiento ingresos gravados y exentos.

Fuente: Chávez (2018). 
Mercedes Elizabeth Cárdenas-Pañi; Cecilia Ivonne Narváez-Zurita; Juan Carlos Erazo-Álvarez; Mireya Magdalena Torres-Palacios

Así mismo, la normativa tributaria toma en cuenta la diferencia entre los gastos deducibles y no deducibles, puesto que tienen tratamiento diferenciado al realizar la conciliación tributaria. En lo que respecta a los gastos deducibles, estos tienen relación directa con la actividad económica de la empresa y disminuyen la base de cálculo del impuesto. En su lugar, los gastos no deducibles, se consideran innecesarios para obtener, mantener o mejorar los ingresos de la empresa, por otro lado, puede tratarse de gastos que excedieron los límites permitidos por la LORTI, por estas situaciones, los gastos no deducibles incrementan la base de cálculo del impuesto (Dirección Nacional Jurídica (DNJ), 2016). Al realizar la conciliación tributaria se debe identificar ingresos gravados, ingresos exentos, gastos deducibles, gastos no deducibles y beneficios tributarios que son otorgados por el gobierno a un sector económico específico, ya que estos modifican la base cálculo del impuesto a la renta (Encalada, Narváez, \& Erazo, 2020).

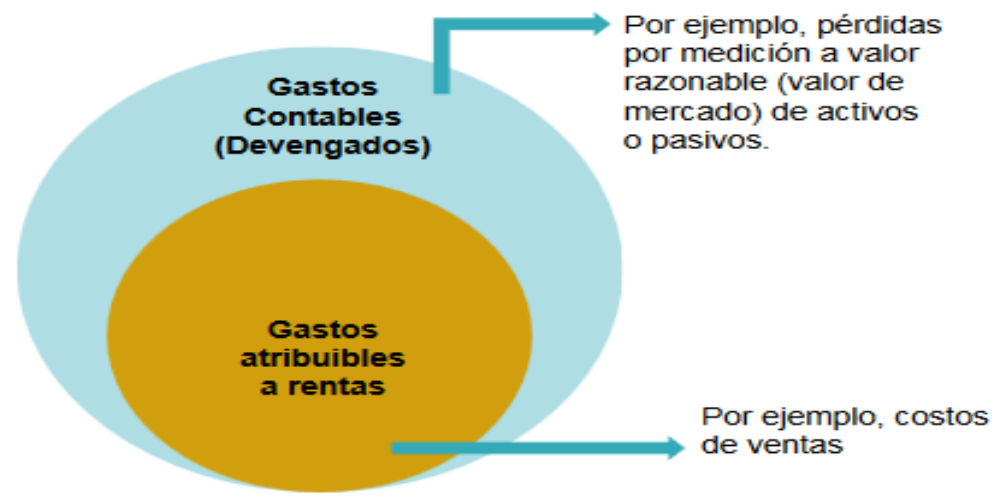

Figura 3. Reconocimiento de gastos deducibles.

Fuente: Chávez (2018).

Por otro lado, (Tobar \& Medina, 2018) y (Condo-Daquimela \& Cobos-Pesántez, 2016), concuerdan en la necesidad de identificar y clasificar los ingresos y gastos en base a la normativa contable vigente en el pais, pudiendo de este modo, obtener la base de 


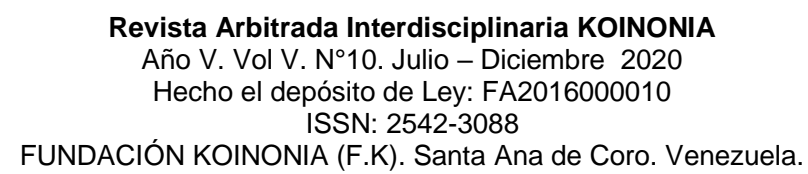

Mercedes Elizabeth Cárdenas-Pañi; Cecilia Ivonne Narváez-Zurita; Juan Carlos Erazo-Álvarez; Mireya Magdalena Torres-Palacios

cálculo del impuesto a la renta, aplicar la tarifa impositiva, y por último determinar el impuesto causado en el ejercicio contable.

\section{El impuesto a las ganancias según la NIC 12}

Las NIIF establecen las pautas para determinar el impuesto causado o también conocido como impuesto a las ganancias contenido dentro de la NIC 12 y la sección 29 de la NIIF para PYMES (Consejo de Normas Internacionales de Contabilidad (IASB), 2009) donde se indican los procedimientos y parámetros para identificar si se trata de un impuesto corriente o diferido y dar un tratamiento adecuado a cada caso. Es importante que las empresas conozcan las consecuencias fiscales de los hechos económicos registrados a lo largo del ejercicio económico, pues éstos se liquidarán en el corto o largo plazo y afectarán las ganancias fiscales.

Por esto, es necesario identificar los impuestos corrientes y los impuestos diferidos en el periodo contable. La sección 29 de la NIIF para PYMES (Consejo de Normas Internacionales de Contabilidad IASB, 2009) muestra la medición y reconocimiento de estos dos tipos de impuestos. Los impuestos corrientes pueden ser:

1. Activo por impuestos corrientes: se recuperará el importe pagado de periodos anteriores con el uso del crédito tributario.

2. Pasivo por impuestos corrientes: se genera al liquidar el impuesto a las ganancias y da como resultado un impuesto a pagar.

Los impuestos diferidos se clasifican en:

1. Activos por impuestos diferidos: esperan a que disminuyan las ganancias fiscales en el futuro, ya sea por pérdidas fiscales o créditos tributarios no utilizados.

2. Pasivos por impuestos diferidos: incrementarán las ganancias fiscales en el futuro.

Los impuestos diferidos se podrán recuperar o pagar en periodos posteriores ya sea cuando se recuperen o liquiden sus activos o pasivos, fiscalmente estos impuestos se 


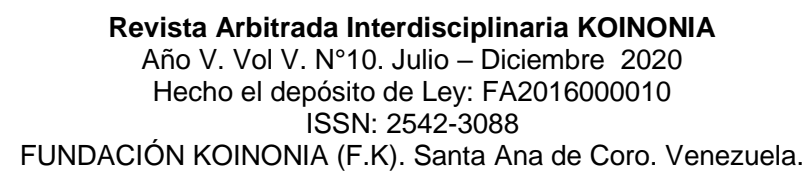

Mercedes Elizabeth Cárdenas-Pañi; Cecilia Ivonne Narváez-Zurita; Juan Carlos Erazo-Álvarez; Mireya Magdalena Torres-Palacios

utilizarán en el momento que se generé un impuesto a pagar o para compensar una pérdida fiscal. Los métodos para el registro de los activos y pasivos diferidos son:

1. El método del pasivo o tradicional (MPBB): hace referencia al impuesto diferido que nace de los resultados obtenidos de la empresa, estos se registrarán en el activo o pasivo diferido según corresponda, compensándose a medida que se devenguen.

2. El método del diferido o de estado de resultados: considera los impuestos diferidos en el resultado, los mismos se reversarán únicamente en el resultado y no se consideran en el activo o pasivo diferido.

En este sentido, (Erazo-Andrade, 2013); (Niño \& Bermúdez, 2016) y (Meneses, Rodriguez, \& Velandia, 2016) coinciden en usar el método tradicional para registrar los impuesto diferidos, ya que se encarga de reflejar los impuestos diferidos generados ya sea como pasivos, (deben ser pagados) o como activos (deben ser recuperados) en la contabilidad de la empresa.

\section{Diferencias entre la base contable y la base fiscal}

Para la determinación del impuesto a las ganancias se realiza la conciliación tributaria, ya que ésta refleja diferencias entre la base contable y la base fiscal. La base contable para la liquidación de impuestos está dada por los ingresos contables menos los gastos contables y la base fiscal se obtiene de los ingresos fiscales menos los gastos fiscales, aquí se puede identificar que no todos los ingresos y gastos que genera la empresa son considerados como ingresos o gastos fiscales, en consecuencia, surgen las diferencias temporarias por impuestos diferidos (Romero, Hansen-Holm, \& Hansen-Holm, 2019) y (Palacios \& Peréz , 2016). Las diferencias temporarias son oposiciones entre el importe en libros y la base fiscal, ya que algunas cuentas de ingreso o gasto afectan a los saldos de los activos y pasivos, tal como se puede observar en la figura 4 (Consejo de Normas Internacionales de Contabilidad IASB, 2009). 
Mercedes Elizabeth Cárdenas-Pañi; Cecilia Ivonne Narváez-Zurita; Juan Carlos Erazo-Álvarez; Mireya Magdalena Torres-Palacios

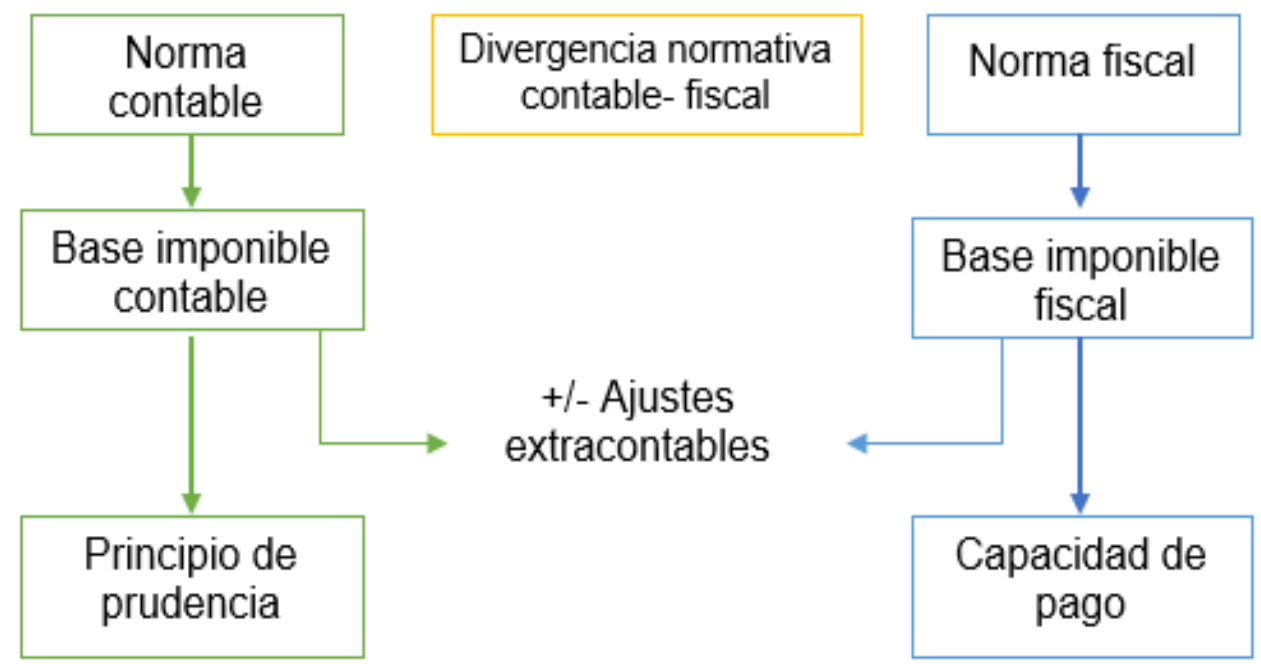

Figura 4. Diferencias entre la base contable y la base fiscal.

Con referencia a las diferencias temporarias estas pueden ser imponibles y deducibles como se puede observar en la figura 5: Las diferencias temporarias imponibles son diferencias entre la base fiscal y la base contable, se reconocen en el resultado integral del periodo corriente dando lugar a cantidades imponibles, los activos por diferencias temporarias imponibles serán recuperados en periodos futuros en manera de beneficios económicos, mientras que, el pasivo por diferencias temporarias imponibles se recuperá cuando se liquide una obligación. Mientras que, las diferencias temporarias deducibles suceden cuando los activos se devenguen o el pasivo se liquide (Consejo de Normas Internacionales de Contabilidad IASB, 2009); (Romero-Morán, Hansen-Holm de Armero \& Hansen-Holm de Armero, 2019) y (Sánchez-Castaño, 2018).

\begin{tabular}{|lllll|}
\hline \multicolumn{2}{|c}{ Diferencia de bases } & $\begin{array}{l}\text { Diferencia } \\
\text { temporaria }\end{array}$ & Reconoce: \\
\hline Activo contable & Mayor & Activo tributario & Imponible & Pasivo diferido \\
\hline Activo contable & Menor & Activo tributario & Deducible & Activo diferido \\
\hline Pasivo tributario & Mayor & Pasivo contable & Deducible & Activo diferido \\
\hline Pasivo tributario & Menor & Pasivo contable & Imponible & Pasivo diferido \\
\hline
\end{tabular}

Figura 5. Reconocimiento de activos y pasivos diferidos.

Fuente: Villegas-Escobar (2018). 


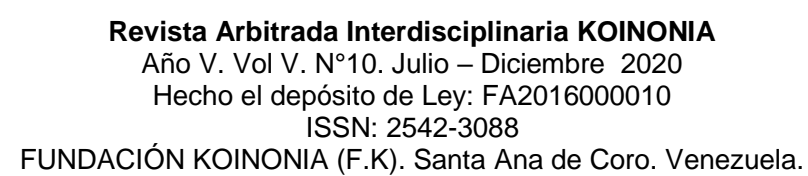

Mercedes Elizabeth Cárdenas-Pañi; Cecilia Ivonne Narváez-Zurita; Juan Carlos Erazo-Álvarez; Mireya Magdalena Torres-Palacios

En efecto, la conciliación tributaria nace de las diferencias entre la base contable y la base fiscal. Contablemente todas las erogaciones de recursos económicos de la empresa son consideradas como gastos, sin embargo, fiscalmente, no todos los gastos, son deducibles para la obtención de la base imponible al liquidar el impuesto a las ganancias. Al hacer esta diferenciación el gobierno como los empresarios siempre han realizado un esfuerzo para llegar a un punto de equilibrio sobre los impuestos que el Estado necesita para atender las necesidades de la colectividad y la estabilidad económica de las empresas, por lo que las leyes se han ido modificando en beneficio de las necesidades, tanto del gobierno, como de los empresarios.

Al respecto, (García-Restrepo, 2009) y (Torres, 2011) afirman que para identificar las diferencias temporarias se deben reconocer primero las diferencias entre la base contable y la base fiscal. Luego se procede a contabilizar las diferencias encontradas mendiante la aplicación del médodo tradicional. Una vez contabilizadas las diferencias se puede indicar que no existe discrepancia entre estas dos bases (contable y fiscal), por lo que se obtendría la base global de cálculo del impuesto a la renta. Cabe recalcar que el tratamiento de los impuestos diferidos nace de la diferencia entre la base contable y la base fiscal y se liquida cuando estos se compensen o devenguen.

\section{MÉTODO}

Esta investigación fue de tipo no experimental, se revisó información referente al cumplimiento de la normativa tributaria y contable de la empresa APC Tecnología. El enfoque fue de tipo descriptivo, los instrumentos utilizados para recabar información fueron la encuesta y la ficha documental, mediante la cual se analizó la información financiera proporcionada por la empresa, este análisis determinó la base contable y tributaria aplicada en la elaboración de la conciliación del impuesto a las ganancias y el tratamiento contable de los impuestos diferidos (Calle-Calle, Narváez-Zurita \& ErazoÁlvarez, 2019). 


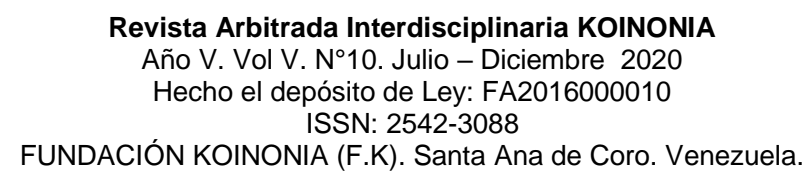

Mercedes Elizabeth Cárdenas-Pañi; Cecilia Ivonne Narváez-Zurita; Juan Carlos Erazo-Álvarez; Mireya Magdalena Torres-Palacios

\section{RESULTADOS}

Entre los principales resultados se determinó lo siguiente:

Aplicación de NIIF: La compañía ha cumplido con las disposiciones del órgano de control (SUPERCIAS), aplicando las NIIF en la elaboración de sus estados financieros.

Políticas contables: las políticas contables sirven como guía para la preparación de los estados financieros, la compañía no cuenta con políticas contables establecidas, los registros contables se realizaron acorde al criterio del contador.

Preparación de estados financieros: los estados financieros son de responsabilidad del contador, estos se han elaborado bajo la normativa contable (NIIF) y normativa tributaria vigente.

Divergencia entre la política contable y tributaria: cuando existe contraposición entre la normativa contable y tributaria, la empresa aplica la normativa contable.

Conciliación del impuesto a las ganancias: al concluir el ejercicio fiscal contablemente se establece discrepancias entre la base contable y tributaria debido a la aparición de diferencias temporarias ya sean estas imponibles o deducibles, por esta situación se realizan ajustes a los estados financieros, surgiendo los impuestos diferidos. La empresa ha realizado de manera adecuada estos ajustes.

Tratamiento de los impuestos diferidos: los impuestos diferidos surgen al momento de reconocer los activos y pasivos a lo largo del ejercicio contable y se evidencia en la conciliación tributaria. En este caso, los impuestos diferidos en la empresa son registrados en la contabilidad y luego se ajustan a la normativa tributaria. La empresa aplica los impuestos diferidos desde el año 2017 por exigencia de auditoria externa.

Aporte de los impuestos diferidos: los impuestos diferidos en la contabilidad de la empresa proporcionan información financiera real y fiable para los usuarios internos y externos. Los impuestos diferidos son extensos y complejos, sin embargo, por obligatoriedad se han aplicado en la medida de lo posible. 
Revista Arbitrada Interdisciplinaria KOINONIA

Año V. Vol V. №10. Julio - Diciembre 2020

Hecho el depósito de Ley: FA2016000010

ISSN: 2542-3088

FUNDACIÓN KOINONIA (F.K). Santa Ana de Coro. Venezuela.

Mercedes Elizabeth Cárdenas-Pañi; Cecilia Ivonne Narváez-Zurita; Juan Carlos Erazo-Álvarez; Mireya

Magdalena Torres-Palacios

\section{PROPUESTA}

De acuerdo a los resultados obtenidos en la investigación se determinó la dificultad de identificar las diferencias entre la normativa contable y tributaria, por lo que se propone una guía (figura 6) para liquidar el impuesto a las ganancias al finalizar el ejercicio económico.

La guía propuesta incluye cinco fases para la conciliación tributaria del impuesto a las ganancias, bajo la normativa fiscal y normativa contable.

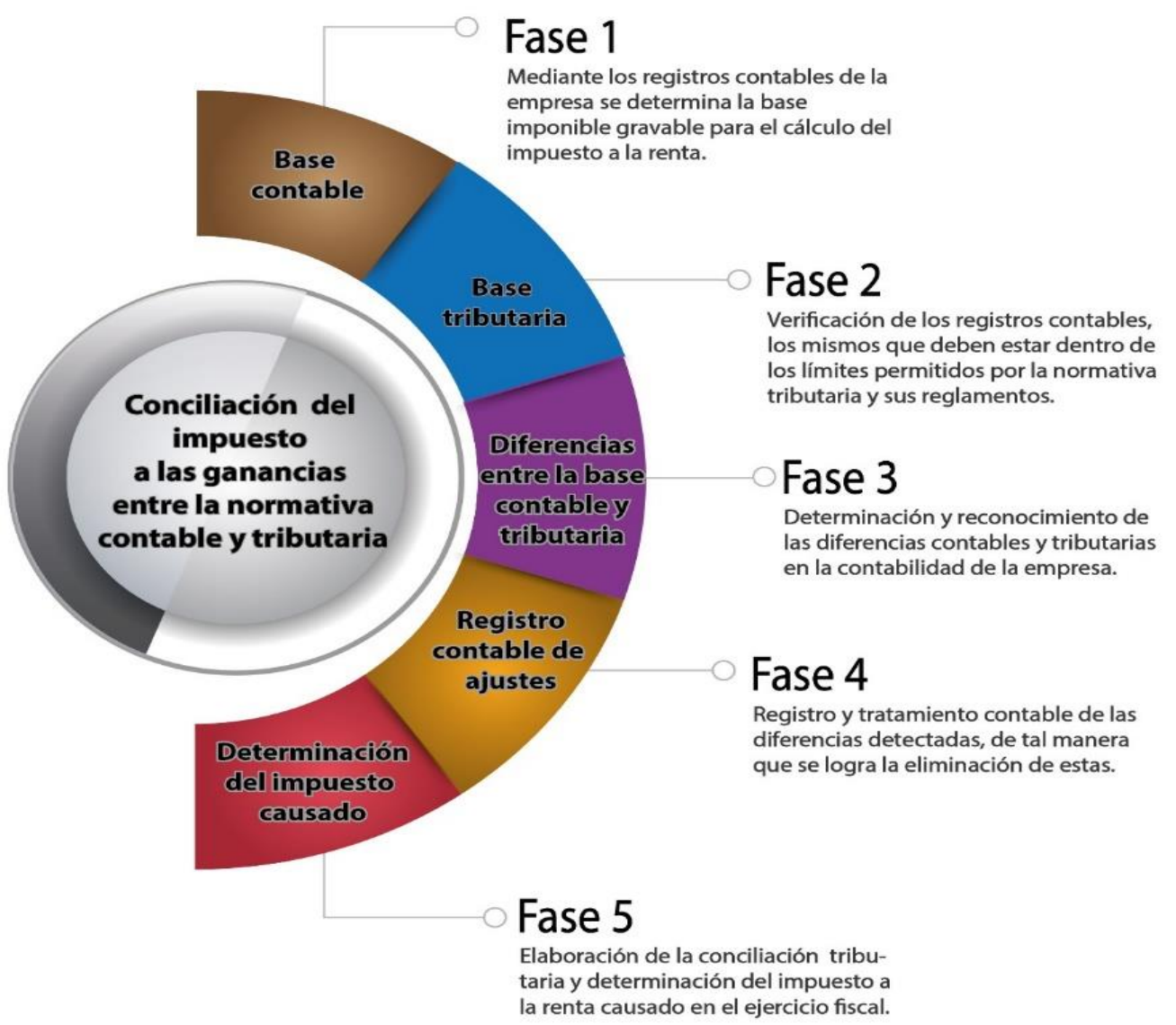

Figura 6. Guía para la conciliación de impuestos a las ganancias un estudio bajo la normativa fiscal y normativa contable. 
Revista Arbitrada Interdisciplinaria KOINONIA

Año V. Vol V. N¹0. Julio - Diciembre 2020

Hecho el depósito de Ley: FA2016000010

ISSN: 2542-3088

FUNDACIÓN KOINONIA (F.K). Santa Ana de Coro. Venezuela.

Mercedes Elizabeth Cárdenas-Pañi; Cecilia Ivonne Narváez-Zurita; Juan Carlos Erazo-Álvarez; Mireya Magdalena Torres-Palacios

\section{Fase 1: Base contable}

La empresa registra las distintas transacciones diarias en base a las NIIF; al concluir el período fiscal que va desde el 1 de enero al 31 de diciembre de cada año, se obtiene la base para el cálculo del impuesto a la renta de la siguiente manera:

$\mathrm{X}=$ Ingresos - Gastos

Donde $\mathrm{X}=$ Base imponible para el cálculo de impuesto a la Renta.

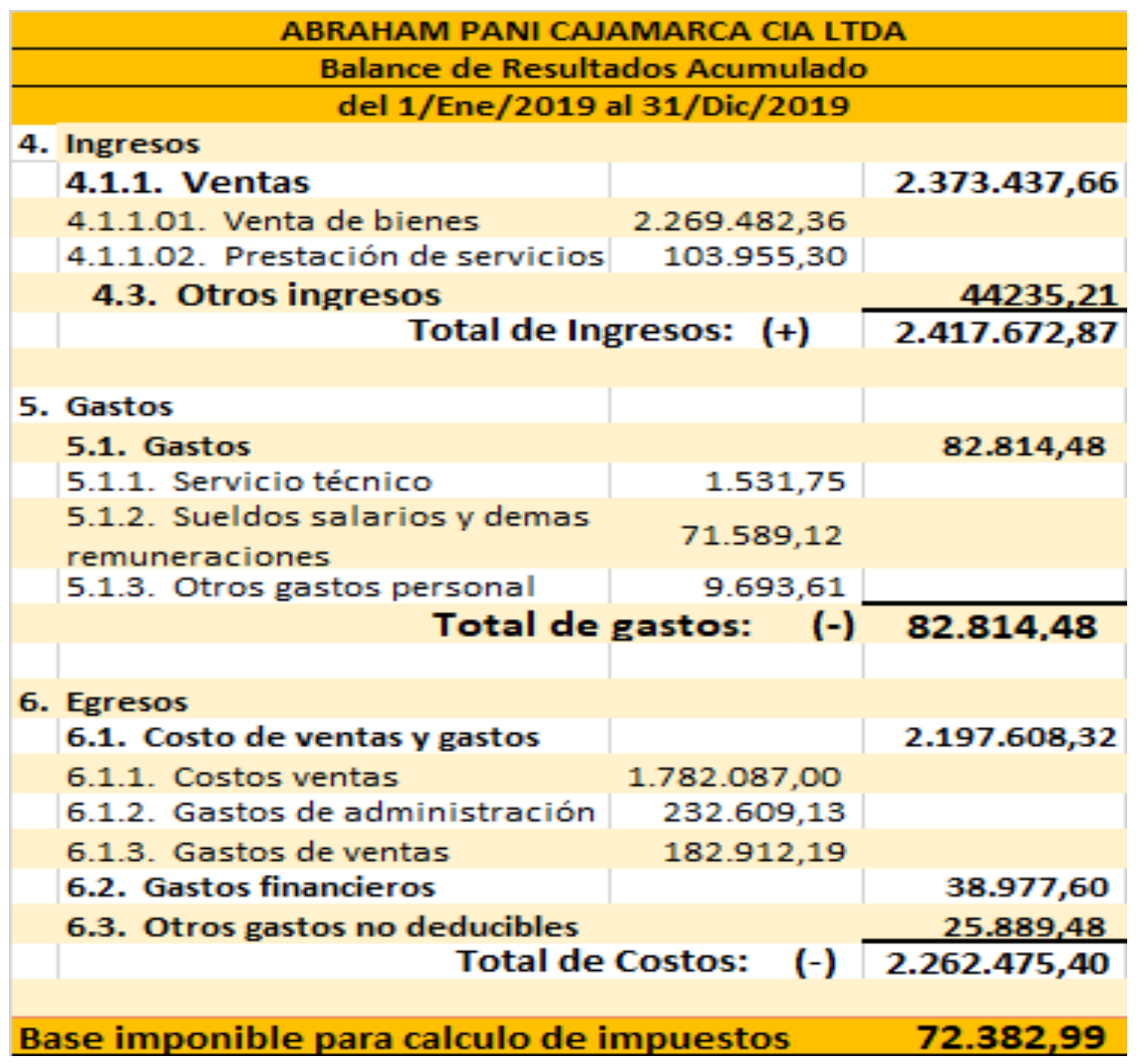

Figura 7. Determinación de base imponible contable.

Fuente: Los datos se extrajeron de los estados financieros de la Compañía ACP Teconología.

\section{Fase 2: Base tributaria}

En esta fase se busca determinar la base imponible para el cálculo del impuesto a la renta obtenida de la contabilidad. Los problemas presentados se deben a la falta de 


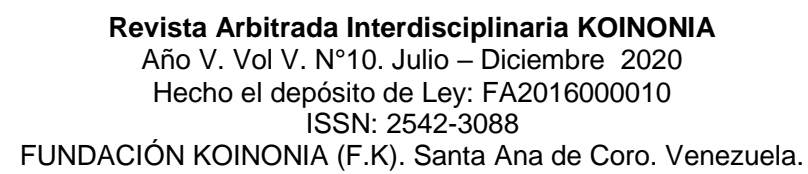

Mercedes Elizabeth Cárdenas-Pañi; Cecilia Ivonne Narváez-Zurita; Juan Carlos Erazo-Álvarez; Mireya Magdalena Torres-Palacios

total aceptación por la administración tributaria, ya que no todos los ingresos y gastos que se encuentra registrados contablemente, son aceptados por la normativa tributaria vigente.

Las principales diferencias tributarias están dadas por: ingresos exentos, gastos no deducibles, deducciones especiales, entre otros, las mismas se encuentran detalladas en la LORTI y sus respectivos reglamentos. Al realizar la conciliación tributaria las principales diferencias encontradas son:

1. Rentas exentas.

2. Gastos no deducibles temporarios.

3. Gastos no deducibles permanentes.

Al finalizar el ejercicio económico anual las empresas elaboran la conciliación entre la base contable y la base fiscal. Contablemente esta comparación se realiza aplicando el método del pasivo basado en el balance (MPBB).

Al aplicar el MPBB se refleja en la contabilidad los activos o pasivos diferidos que se generaron o reversaron en el ejercicio económico. Fiscalmente se lo realiza mediante la conciliación tributaria en la que se identifica los rubros que incrementan o disminuyen la base imponible para el cálculo del impuesto a la renta. Una vez identificadas las divergencias de la base de cálculo del impuesto a la renta que se obtiene de la contabilidad y de la aplicación de la normativa tributaria se procede a realizar ajustes extracontables de esta manera se eliminan las diferencias entre la base contable y tributaria, entonces se puede realizar el cálculo del impuesto causado corriente y diferido mediante la aplicación de la tarifa impositiva correspondiente.

\section{Fase 3: Diferencias entre la base contable y tributaria}

Al realizar la conciliación del impuesto a las ganancias se destaca que los rubros más complejos de identificar son los gastos no deducibles, estos se puede originar por:

1. Gastos sin sustento tributario.

2. Gastos sin aplicación de retención. 
Mercedes Elizabeth Cárdenas-Pañi; Cecilia Ivonne Narváez-Zurita; Juan Carlos Erazo-Álvarez; Mireya Magdalena Torres-Palacios

3. Multas pagadas.

4. Falta de bancarización.

5. Exceso en depreciaciones (depreciación acelerada) sin autorización por la administración tributaria.

6. Exceso en límites de gastos permitidos por la normativa tributaria vigente.

7. Erogaciones de dinero que no están relacionados con el giro del negocio. Entre otras situaciones.

Los gastos no deducibles generan diferencias temporarias, siendo necesario identificarlos correctamente y realizar los ajustes extracontables de una manera adecuada (figura 8):

\begin{tabular}{l|ll}
$\begin{array}{l}\text { Gasto no deducible } \\
\text { Se puede recuperar }\end{array}$ & $\begin{array}{l}\text { Tipo gasto no } \\
\text { deducible }\end{array}$ & \multicolumn{1}{c}{$\begin{array}{c}\text { Ejemplo } \\
\text { en periodos } \\
\text { contables } \\
\text { posteriores. }\end{array}$} \\
$\begin{array}{lll}\text { No se recuperan en } \\
\text { periodos contables } \\
\text { posteriores. }\end{array}$ & Temporarios & $\begin{array}{l}\text { Provisiones por } \\
\text { jubilación patronal y } \\
\text { desahucios. } \\
\text { Provisión por deterioro }\end{array}$ \\
\hline
\end{tabular}

Figura 8. Reconocimiento de diferencias temporarias en gastos no deducibles

\section{Diferencias permanentes y temporarias}

1. Las diferencias temporarias permanentes no generan impuestos diferidos ya que se liquidan en el periodo contable que se originan.

2. Las diferencias temporarias generan impuestos diferidos al ser recuperados 0 liquidados en periodos contables futuros.

Como se puede observar en la figura 9 , el asiento contable 1 registra las diferencias temporarias que afectan a las cuentas del gasto y del pasivo, generándose impuestos diferidos al reversar la cuenta del pasivo en ejercicios económicos posteriores hasta 
Mercedes Elizabeth Cárdenas-Pañi; Cecilia Ivonne Narváez-Zurita; Juan Carlos Erazo-Álvarez; Mireya Magdalena Torres-Palacios

que se liquide. Mientras que las diferencias temporarias permanentes como se puede apreciar en el asiento 2 afecta al gasto y al activo circulante y no tienen incidencia en períodos contables posteriores.

\begin{tabular}{|c|c|c|}
\hline CUENTAS & DEBE & HABER \\
\hline \multicolumn{3}{|l|}{1} \\
\hline Gasto provisión de jubilación patronal & $10.329,26$ & \\
\hline Cuentas por pagar jubilación patronal & & 10329,26 \\
\hline \multicolumn{3}{|c|}{ P/R: Contabilización de diferencias temporarias } \\
\hline \multicolumn{3}{|c|}{2} \\
\hline $\begin{array}{l}\text { Gastos no deducibles/pago de multas de } \\
\text { tránsito }\end{array}$ & 106,69 & \\
\hline Bancos & & 106,69 \\
\hline
\end{tabular}

Figura 9. Contabilización de diferencias temporarias y diferencias temporarias permanentes.

Fuente: estados financieros de la Compañía ACPTecnología.

Al concluir el ejercicio económico la empresa obtiene la utilidad contable, a partir de esta base imponible se realiza los ajustes extracontables mediante la aplicación de la NIC 12 y la sección 29 de la NIIF para PYMES, de esta manera se eliminan las diferencias entre la base contable y la base fiscal. Los ajustes extracontables únicamente se registrarán cuando existan diferencias temporarias deducibles, éstas afectan, tanto a los activos o pasivos diferidos, como a la conciliación tributaria.

Mientras que, las diferencias temporarias ya se encuentran registradas en la contabilidad afectando únicamente a la conciliación tributaria. Una vez reconocidas las diferencias temporarias es necesario que se identifique si estas se registran como activos o pasivos diferidos como se puede observar en la figura 10. 
Revista Arbitrada Interdisciplinaria KOINONIA

Año V. Vol V. №10. Julio - Diciembre 2020

Hecho el depósito de Ley: FA2016000010

ISSN: 2542-3088

FUNDACIÓN KOINONIA (F.K). Santa Ana de Coro. Venezuela.

Mercedes Elizabeth Cárdenas-Pañi; Cecilia Ivonne Narváez-Zurita; Juan Carlos Erazo-Álvarez; Mireya Magdalena Torres-Palacios

\begin{tabular}{lllll}
\multicolumn{2}{c}{ Diferencia de bases } & $\begin{array}{l}\text { Diferencia } \\
\text { temporaria }\end{array}$ & Se reconoce \\
\hline Activo contable & Mayor Activo tributario & Imponible & Pasivo diferido \\
\hline Activo contable & Menor & Activo tributario & Deducible & Activo diferido \\
\hline Pasivo tributario & Mayor & Pasivo contable & Deducible & Activo diferido \\
\hline Pasivo tributario & Menor & Pasivo contable & Imponible & Pasivo diferido \\
\hline
\end{tabular}

Figura 10. Reconocimiento de activos y pasivo diferidos.

Dado lo anterior, se debe anotar que las diferencias temporarias imponibles generan pasivos diferidos y las diferencias temporarias deducibles generan activos por impuestos diferidos.

\section{Fase 4: Registro contable de ajustes}

A continuación, en la figura 11 se presentan los distintos escenarios para el registro de los asientos contables de las diferencias temporarias deducibles.

\begin{tabular}{|c|c|c|c|}
\hline Concepto & Cuenta & Debe & Haber \\
\hline Diferencias temporarias & Activo & $x \times X X$ & \\
\hline Ingresos por diferencias temporarias & Ingreso & & $x \times X X$ \\
\hline \multicolumn{4}{|l|}{ P/R: Generación activo diferido } \\
\hline Gas to por impues to diferido U ORI & Gasto & $x \times x x$ & \\
\hline Diferencias temporarias & Activo & & $X X X X$ \\
\hline \multicolumn{4}{|c|}{ P/R: Reversión activo por impuesto diferido } \\
\hline Gasto por impues to diferido U ORI & Gasto & $x \times x x$ & \\
\hline Pasivo por impuesto diferido & Pasivo & & $x \times x x$ \\
\hline \multicolumn{4}{|l|}{ P/R: Generación pasivo diferido } \\
\hline Pasivo por impues to diferido & Pasivo & $X X X X$ & \\
\hline Ingresos por diferencias temporarias & Ingreso & & $X X X X$ \\
\hline P/R: Reversión pasivo por impuestos & los & & \\
\hline
\end{tabular}

Figura 11. Contabilización de impuestos diferidos 
Revista Arbitrada Interdisciplinaria KOINONIA

Año V. Vol V. N¹0. Julio - Diciembre 2020

Hecho el depósito de Ley: FA2016000010

ISSN: 2542-3088

FUNDACIÓN KOINONIA (F.K). Santa Ana de Coro. Venezuela.

Mercedes Elizabeth Cárdenas-Pañi; Cecilia Ivonne Narváez-Zurita; Juan Carlos Erazo-Álvarez; Mireya Magdalena Torres-Palacios

La figura 12 muestra los datos obtenidos de la empresa, a partir de los cuales se realizan los asientos contables correspondientes, considerando los datos adicionales.

La empresa APC Tecnología al concluir las operaciones en el año 2019 obtuvo los siguientes resultados.

Ingresos Operacionales

(-) Costos Operacionales

(=) Utilidad Bruta

(-) Gastos Operacionales

(=) Utilidad Operacional

(+) Ingresos No Operacionalt

(=) Utilidad Contable
2.380.707,49

1.782.087, 00

$\mathbf{5 9 8 . 6 2 0 , 4 9}$

$563.202,88$

$35.417,61$

$36.965,38$

72.382,99

Como gastos no deducibles registraron estos rubros:

Concepto

6.1.2.06.006 DONACIONES

6.3.002 INTERESES Y MULTAS

6.3.003 OTROS GASTOS NO DEDUCIBLES

6.3.006 DETERIORO DE ANTICIPO PROVEEDORES

6.3.004 GASTO PROVISION JUBILACION

6.3.005 GASTO PROVISION DESAHUCIO
Monto

156,96

106,69 Permanente

$1.561,32$ Permanente

12.052,77 Permanente

10.329,26 Deducible

$1.824,30$ Deducible

\section{Datos adicionales:}

- El estudio actuarial generó un gasto para el año 2019 por provisión de jubiliación patronal por $\$ 10.329,26$ y por provisión de desahucio por $\$ 1.824,30$.

Contabilización:

\begin{tabular}{|c|c|c|c|}
\hline CUENTAS & & DEBE & HABER \\
\hline \multicolumn{4}{|l|}{1} \\
\hline Gasto provisión de jubilación patronal & GASTO & 10329,3 & \\
\hline Cuentas por pagar jubilación patronal & PASIVO & & 10329,26 \\
\hline \multicolumn{4}{|c|}{ P/R: Por provisión de jubilación patronal } \\
\hline \multicolumn{4}{|c|}{2} \\
\hline Gasto provisión de desahucio & GASTO & 1824,30 & \\
\hline Cuentas por pagar desahucio & PASIVO & & 1824,30 \\
\hline
\end{tabular}

Figura 12. Contabilización de las diferencias temporarias deducibles. Fuente: APC Tecnología. 
Revista Arbitrada Interdisciplinaria KOINONIA

Año V. Vol V. №10. Julio - Diciembre 2020

Hecho el depósito de Ley: FA2016000010

ISSN: 2542-3088

FUNDACIÓN KOINONIA (F.K). Santa Ana de Coro. Venezuela.

Mercedes Elizabeth Cárdenas-Pañi; Cecilia Ivonne Narváez-Zurita; Juan Carlos Erazo-Álvarez; Mireya Magdalena Torres-Palacios

Las diferencias temporarias encontradas se registran en la contabilidad de la empresa (figura 12), de esta manera se eliminan las disconformidades entre la normativa contable y la normativa tributaria. Los impuestos diferidos generados por las diferencias temporarias se esperan recuperar en periodos contables posteriores registrándolos en la conciliación tributaria y obteniendo la base imponible para la aplicación de la tarifa impositiva del impuesto a la renta.

\section{Fase 5: Determinación del impuesto causado}

A continuación, en la figura 13, se muestra un modelo de conciliación tributaria.

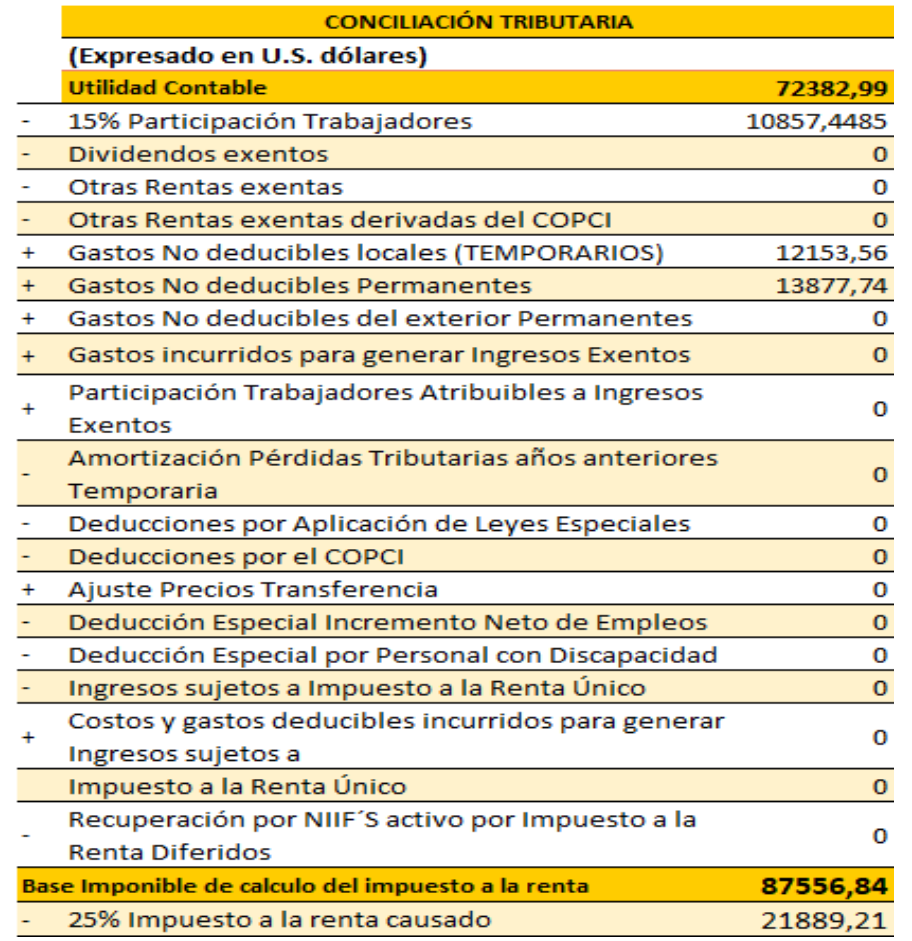

Figura 13. Conciliación del impuesto a las ganancias o conciliación tributaria. Fuente: estados financieros de la Compañía ACP Tecnología. 
Mercedes Elizabeth Cárdenas-Pañi; Cecilia Ivonne Narváez-Zurita; Juan Carlos Erazo-Álvarez; Mireya Magdalena Torres-Palacios

Como se puede observar en la figura 14, la base imponible para el cálculo del impuesto a la renta se incrementa, al existir gastos no deducibles, tanto por diferencia temporaria como por diferencia temporaria deducible, acrecentando el impuesto a la renta causado. Identificados los gastos no deducibles permanentes y temporarios se puede establecer el impuesto a la renta a recuperar en periodos contables posteriores, considerando que los gastos no deducibles temporarios son los únicos a recuperar.

Gasto por provision para el año 2019

\begin{tabular}{lr}
\hline Gasto provisión jubilacion patronal & 10329,26 \\
\hline Gasto provisión de desahucio & 1824,3 \\
\hline Total gasto por provision año 2019 & 12153,56 \\
\hline $\begin{array}{l}\text { Impuesto a la renta causado por } \\
\text { generacion de impuestos diferidos }\end{array}$ \\
$\begin{array}{l}\text { (aplica tarifa impuesto a la renta 25\%) } \\
\text { La tarifa que se aplica para la obtención del impuesto } \\
\text { diferido es la que corresponde en al año fiscal en el que }\end{array}$ \\
$\begin{array}{l}\text { se realiza el calculo. }\end{array}$
\end{tabular}

Figura 14. Cálculo las diferencias temporarias a recuperar en periodos contables posteriores. 
Revista Arbitrada Interdisciplinaria KOINONIA

Año V. Vol V. N¹0. Julio - Diciembre 2020

Hecho el depósito de Ley: FA2016000010

ISSN: 2542-3088

FUNDACIÓN KOINONIA (F.K). Santa Ana de Coro. Venezuela.

Mercedes Elizabeth Cárdenas-Pañi; Cecilia Ivonne Narváez-Zurita; Juan Carlos Erazo-Álvarez; Mireya Magdalena Torres-Palacios

La generación de diferencias temporarias deducibles se tributa de manera anticipada y se recupera en periodos contables posteriores a la liquidación de la obligación.

\begin{tabular}{|c|c|c|}
\hline \multicolumn{3}{|c|}{ Conciliación tributaria } \\
\hline & \multicolumn{2}{|l|}{ (Expresado en U.S. dólares) } \\
\hline & Utilidad Contable & $\mathbf{X X X}$ \\
\hline- & 15\% Participación Trabajadores & $\mathrm{XXX}$ \\
\hline+ & Gastos No deducibles locales (TEMPORARIOS) & $X X X$ \\
\hline+ & Gastos No deducibles Permanentes & $X X X$ \\
\hline & $\begin{array}{l}\text { Recuperación por NIIF'S activo por Impuesto a la } \\
\text { Renta Diferidos }\end{array}$ & $\mathrm{XXX}$ \\
\hline & Base Imponible de calculo del impuesto a la renta & $\mathbf{X X X}$ \\
\hline- & $25 \%$ Impuesto a la renta causado & $\mathrm{XXX}$ \\
\hline \multicolumn{3}{|c|}{ Determinación utilidad contable del ejercicio. } \\
\hline & Utilidad Contable & $\mathbf{x X X}$ \\
\hline - & 15\% Participación Trabajadores & $\mathrm{XXX}$ \\
\hline & $25 \%$ Impuesto a la renta causado & $X X X$ \\
\hline - & $5 \%$ Reserva Legal & $x X X$ \\
\hline & Impuesto por diferencias temporarias & $X X X$ \\
\hline & ilidad del ejercicio & $\mathbf{x x x}$ \\
\hline
\end{tabular}

Contabilización de conciliación tributaria por recuperación de impuestos diferidos

\begin{tabular}{|c|c|c|c|}
\hline Cuentas & & Debe & Haber \\
\hline \multicolumn{4}{|l|}{1} \\
\hline Resultados del ejercicio & Patrimonio & $x x x$ & \\
\hline Participación trabajadores & Pasivo & $x x x$ & \\
\hline Impuesto a la renta por pagar & Pasivo & & $x x x$ \\
\hline Reserva legal & Patrimonio & & $x x x$ \\
\hline $\begin{array}{l}\text { Diferencias temporatias por } \\
\text { impuestos diferidos }\end{array}$ & Activo & & $x X X$ \\
\hline P/R: Registro de conciliación tri & ria & & \\
\hline
\end{tabular}

Figura 15. Recuperación de impuestos diferidos en periodos posteriores. 
Revista Arbitrada Interdisciplinaria KOINONIA

Año V. Vol V. N¹0. Julio - Diciembre 2020

Hecho el depósito de Ley: FA2016000010

ISSN: 2542-3088

FUNDACIÓN KOINONIA (F.K). Santa Ana de Coro. Venezuela.

Mercedes Elizabeth Cárdenas-Pañi; Cecilia Ivonne Narváez-Zurita; Juan Carlos Erazo-Álvarez; Mireya Magdalena Torres-Palacios

Obtenidos todos los rubros que intervienen en la liquidación de la utilidad del ejercicio, se puede observar el impacto del impuesto diferido generado por las diferencias temporarias deducibles (figura 16).

\begin{tabular}{|c|c|}
\hline Utilidad Contable & $72.382,99$ \\
\hline 15\% Participación Trabajadores & $10.857,45$ \\
\hline $25 \%$ Impuesto a la renta causado & $21.889,21$ \\
\hline - 5\% Reserva Legal & $1.981,82$ \\
\hline + Impuesto por diferencias & $3.038,39$ \\
\hline Utilidad del ejercicio & $40.692,90$ \\
\hline
\end{tabular}

Contabilización de conciliación tributaria.

\begin{tabular}{|c|c|c|c|}
\hline Cuentas & & Debe & Haber \\
\hline \multicolumn{4}{|l|}{1} \\
\hline $\begin{array}{l}\text { Diferencias temporatias por } \\
\text { impuestos diferidos }\end{array}$ & Activo & $3.038,39$ & \\
\hline Resultados del ejercicio & Patrimonio & $31.690,09$ & \\
\hline Participación trabajadores & Pasivo & & 10857,45 \\
\hline Impuesto a la renta por pagar & Pasivo & & 21889,21 \\
\hline Reserva legal & Patrimonio & & 1981,82 \\
\hline \multicolumn{4}{|c|}{ P/R: Registro de conciliación tributaria } \\
\hline \multicolumn{4}{|c|}{ Mayor contable de utilidad del ejercicio } \\
\hline \multicolumn{4}{|l|}{ Cuenta: Resultado del ejercicio } \\
\hline $\begin{array}{l}\text { Descripcion } \\
\end{array}$ & Debe & Haber & Saldo \\
\hline Saldo inicial & & & 0,00 \\
\hline $\begin{array}{l}\text { Cierre de estado de } \\
\text { resultados }\end{array}$ & & $72.382,99$ & $72.382,99$ \\
\hline Registro conciliación tributaria & $31.690,09$ & & $40.692,90$ \\
\hline
\end{tabular}

Figura 16. Liquidación de impuesto del ejercicio.

Se debe proceder de forma obligatoria a la verificación del valor neto realizable (VNR) del inventario existente, debido al deterioro acelerado que sufre este tipo de productos tecnológicos. El tratamiento contable adecuado se encuentra plasmado en la NIC 2 y en el Art. 10 de la LORTI (Ley Orgánica de Régimen Tributario Interno) numeral 1. Contablemente el VNR se registra en el ejercicio económico corriente como deterioro en el inventario, mientras que, la norma tributaria indica, que esta provisión se reconoce como gasto no deducible generando impuestos diferidos y se recuperará al momento de 
Mercedes Elizabeth Cárdenas-Pañi; Cecilia Ivonne Narváez-Zurita; Juan Carlos Erazo-Álvarez; Mireya Magdalena Torres-Palacios

la enajenación o consumo. Por lo tanto, se dejará plasmado los respectivos asientos contables que la empresa puede utilizar cuando realice la provisión por el VNR a los inventarios (figura 17).

Generación de impuestos diferidos por provisión VNR

\begin{tabular}{|c|c|c|}
\hline Cuentas & Debe & Haber \\
\hline \multicolumn{3}{|l|}{1} \\
\hline Pérdida por deterioro en inventarios & $\mathrm{XXX}$ & \\
\hline Deterioro del valor de inventarios & & $X X X X$ \\
\hline \multicolumn{3}{|l|}{ P/R: Registro deterior de inventarios } \\
\hline \multicolumn{3}{|l|}{2} \\
\hline Gasto impuesto a la renta & $\mathrm{XXX}$ & \\
\hline Activo por impuesto diferido & $\mathrm{XXX}$ & \\
\hline Impuesto a la renta corriente & \multicolumn{2}{|c|}{$\mathrm{XXXX}$} \\
\hline \multicolumn{3}{|l|}{ P/R: Registro de impuesto diferido } \\
\hline \multicolumn{3}{|c|}{ Recuperacion de impuesto diferido por provisón del VNR } \\
\hline Cuentas & Debe & Haber \\
\hline \multicolumn{3}{|l|}{1} \\
\hline Gasto impuesto a la renta & $\mathrm{XXX}$ & \\
\hline Activo por impuesto diferido & & $\mathrm{XXX}$ \\
\hline Impuesto a la renta corriente & & $\mathrm{XXX}$ \\
\hline
\end{tabular}

Figura 17. Registro contable de generación y reversión de la provisión por VNR

\section{DISCUSIÓN}

En el Ecuador a partir del año 2010 la SUPERCIAS estableció que la contabilidad de las empresas se lleve bajo las NIIF o las NIIF para PYMES y a la par se aplique la normativa tributaria vigente. Esta transición obliga no solo a las empresas a establecer nuevas políticas y procedimientos, también los órganos de control como la SUPERCIAS y el SRI están obligados a expedir nuevos procedimientos y normativas que aporten al adecuado control financiero de las empresas. 


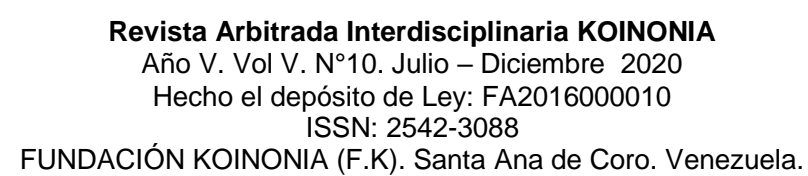

Mercedes Elizabeth Cárdenas-Pañi; Cecilia Ivonne Narváez-Zurita; Juan Carlos Erazo-Álvarez; Mireya Magdalena Torres-Palacios

Las normas que rigen el manejo contable y tributario conjunto de las empresas han generado ciertas divergencias, por una parte, la normativa contable busca demostrar fielmente su situación financiera; mientras que, la normativa tributaria tiene por finalidad determinar el impuesto a las ganancias, estableciendo la base imponible del impuesto a la renta, la misma que está sustentada en la LORTI y sus reglamentos.

Al no existir concordancia entre la norma tributaria y la norma contable se ha buscado un punto de equilibrio denominado impuesto diferido, su objetivo es recuperar los impuestos que las empresas han cancelado por anticipado en periodos económicos posteriores mediante la elaboración de la conciliación tributaria. Los impuestos diferidos no solamente tienen incidencia en la liquidación del impuesto a las ganancias sino también en las cuentas de activo, pasivo, patrimonio, ingresos y gastos; es decir, en la contabilidad íntegra de la empresa. En el caso particular de la empresa APC Tecnología, se evidencia que, al no contar con políticas contables establecidas, la información financiera no refleja fielmente su realidad económica. Por lo tanto, los puntos débiles a reforzar son:

1. Implementación de políticas contables bajo NIIF.

2. Razonabilidad de los inventarios mediante la aplicación de pruebas del VNR que brinde datos reales del valor de los inventarios.

Una de las desventajas de las empresas PYMES, como APC tecnología, es no contar con un departamento de contabilidad capacitado para el manejo de las normativas contables y tributarias vigentes. El adecuado manejo de estas normativas es de vital importancia para la empresa, ya que de esta manera se obtendrá información financiera confiable y normativa tributaria cumplida.

Como objetivo de la investigación se propone una guía para la conciliación del impuesto a las ganancias bajo la normativa fiscal y normativa contable, el fin de esta guía es identificar los impuestos diferidos que nacen de la determinación del impuesto a las ganancias mediante la elaboración de la conciliación tributaria desarrollada dentro del 
Mercedes Elizabeth Cárdenas-Pañi; Cecilia Ivonne Narváez-Zurita; Juan Carlos Erazo-Álvarez; Mireya Magdalena Torres-Palacios

presente documento, en cinco fases, que van desde su nacimiento hasta su recuperación o liquidación.

Mediante la aplicación de esta guía se pretende identificar de manera oportuna los impuestos diferidos, proporcionando a la empresa una herramienta que presente la situación financiera sustentada en la normativa contable y tributaria, así como su reconocimiento inicial, el tratamiento adecuado, recuperación y liquidación de los impuestos diferidos.

\section{FINANCIAMIENTO}

No monetario.

\section{AGRADECIMIENTO}

A la Directiva de la empresa APC Tecnología por apoyar el desarrollo de esta investigación.

\section{REFERENCIAS CONSULTADAS}

Cabrera-Álvarez, P., Narváez-Zurita, C., \& Erazo-Álvarez, J. (2020). Análisis de la cultura tributaria de los consumidores y comerciantes de productos cárnicos en el cantón Santa Isabel, provincia del Azuay. [Analysis of the tax culture of consumers and meat products' merchants in Santa Isabel canton, Azuay province]. Dominio de las ciencias, Vol. 6, №. Extra 1, 341-368

Calle-Calle, A., Narváez-Zurita, C., \& Erazo-Álvarez, J. (2019). Auditoria en prevención de riesgos laborales y salud ocupacional: Procedimiento sistémico aplicado a la empresa Jasetrón. [Audit in prevention of occupational risks and occupational health: Systemic procedure applied to the Jasetron company.]. Revista Arbitrada Interdisciplinaria Koinonía, 4(2), 25-55. http://dx.doi.org/10.35381/r.k.v4i2.466

Campoverde-León, J., \& Castro-Garcés, C. (2016). Diseño de una metodología para la implementación de NIIF 15 en una empresa inmobiliaria en el Ecuador y sus efectos tributarios. [Design of a methodology for the implementation of IFRS 15 in a real estate company and its tax effects in Ecuador.]. Recuperado de https://n9.cl/9jddm 
Revista Arbitrada Interdisciplinaria KOINONIA

Año V. Vol V. N¹0. Julio - Diciembre 2020

Hecho el depósito de Ley: FA2016000010

ISSN: 2542-3088

FUNDACIÓN KOINONIA (F.K). Santa Ana de Coro. Venezuela.

Mercedes Elizabeth Cárdenas-Pañi; Cecilia Ivonne Narváez-Zurita; Juan Carlos Erazo-Álvarez; Mireya Magdalena Torres-Palacios

Chávez, L. (2018). NIIF y tributación: desafíos y oportuniades para las administraciones tributarias. [IFRS and Taxation: challenges and opportunities for tax administrations]. Revista de administración tributaria CIATIAEATIIEF № 43, 6-26. Recuperado de https://n9.cl/98anh

Chávez-Pullas, M. A., \& Herrera-Estrella, M. (2018). Aplicación de las NIIF en Ecuador desde la perspectiva contable positiva. [Application of IFRS in Ecuador from a positive accounting perspective.]. Recuperado de https://n9.cl/exgv

Condo-Daquimela, D., \& Cobos-Pesántez, M. (2016). Incidencia tributaria en la aplicación de las NIC y las NIF para PYMES en la empresa OFFICENTER en la ciudad de Cuenca. [Tax incidence in the application of the IAS and the IFRS for SMEs in the company OFFICENTER in Cuenca city.]. Recuperado de https://n9.cl/2ck6

Cóndor-Paucar, W. (2015). Principales Normas Internacionales de Información Financiera y el impacto en el campo tributario sobre el impuesto a las ganancias en Ecuador.[Main International Financial Reporting Standards and the impact in the tax field on income tax in Ecuador]. Recuperado de https://n9.cl/g8ju

Consejo de Normas Internacionales de Contabilidad (IASB, 2009). Normas Internacionales de Informacion Financiera (NIIF) para Pequeñas y Medianas Entidades (PYMES). [International Financial Reporting Standards (IFRS) for Small and Medium Entities (SMEs)]. Recuperado de https://n9.cl/9yzi

Dirección Nacional Jurídica (DNJ, 2016). Ley Orgánica de Régimen Tributario Interno, LORTI [Organic Law of Internal Tax Regime, OLITR]. Recuperado de https://n9.cl/tzppx

Encalada, D., Narváez, C., \& Erazo, J. (2020). La planificación tributaria, una herramienta útil para la toma de decisiones en las comercializadoras de GLP. [Tax planning, a useful tool for decision-making in LPG traders]. Dominio de las ciencias, Vol. 6, №. Extra 1, 100-126.

Erazo-Andrade, J. (2013). Análisis de la NIC 12 (Impuesto sobre las Ganancias) a la luz de la legislación tributaria vigente Caso Toyota del Ecuador S. A. [Analysis of IAS 12 (Income Tax) in light of the current tax legislation Case Toyota del Ecuador S.A]. Recuperado de https://n9.cl/z1hv

García-Restrepo, J. (2009). El manejo del impuesto diferido en Colombia. [Deferred tax management in Colombia]. Revista Visión Contable, (7), 11-50. Recuperado de https://n9.cl//945 
Revista Arbitrada Interdisciplinaria KOINONIA

Año V. Vol V. N¹0. Julio - Diciembre 2020

Hecho el depósito de Ley: FA2016000010

ISSN: 2542-3088

FUNDACIÓN KOINONIA (F.K). Santa Ana de Coro. Venezuela.

Mercedes Elizabeth Cárdenas-Pañi; Cecilia Ivonne Narváez-Zurita; Juan Carlos Erazo-Álvarez; Mireya Magdalena Torres-Palacios

García-Carvajal, S., \& Dueñas-Casallas, R. (2016). Los aspectos cualitativos en las PYMES y los nuevos retos gerenciales frente a las NIIF. [The qualitative aspects in SMEs and the new management challenges facing IFRS.]. Revista Academia \& Virtualidad 9, 109-120. http://dx.doi.org/10.18359/ravi.2010

Mena, R., Narváez, C. I., \& Erazo, J. C. (2019). La planificación tributaria como herramienta financiera parala optimización de los recursos empresariales. [Tax planning as a financial tool for optimizing business resources]. Visionario Digital, 6-34. https://doi.org/10.33262/visionariodigital.v3i2.1..540

Meneses, M., Rodriguez, L., \& Velandia, J. (2016). Impacto en el reconocimiento, medición y revaluación del impuesto diferido bajo NIC 12 en las propiedades, planta y equipo. [Impact on the recognition, measurement and revaluation of deferred tax under IAS 12 on property, plant and equipment]. Programa de contaduria pública especialización en gestión tributaria. Bogotá, Colombia. Recuperado de https://n9.cl/nj6n

Niño, Z., \& Bermúdez, L. (2016). Efectos fiscales del impuesto diferido NIIF PYMES sección 29 frente a las pérdidas en activos. [Tax effects of deferred tax IFRS SMEs section 29 against losses on assets.]. Recuperado de https://n9.cl/2n9rk

Palacios, J., \& Peréz , M. (2016). Aplicabilidad de la sección 29 de la NIIF para las PYMES "impuesto a las ganancias" a la luz de la normativa tributaria ecuatoriana. [Applying Section 29 Income Tax of the IFRS for SMEs in light of Ecuadorian tax regulations.]. Recuperado de https://n9.cl/nofb

Richardson, A., \& Eberlein, B. (2011). Legitimating Transnational Standard-Setting: The Case of the International Accounting Standards Board. [Legitimación del establecimiento de normas transnacionales: el caso de la Junta de Normas Internacionales de Contabilidad]. Journal of Business Ethics, 217-245. https://doi.org/10.1007/s10551-010-0543-9

Romero-Morán, F., Hansen-Holm de Armero, J. \& Hansen-Holm de Armero, M. (2019). Manual de Obligaciones Tributarias 2019. [Tax obligations manual]. Guayaquil, Ecuador: Hansen Holm \& Co.

Sánchez-Castaño, J. A. (2018). Elementos conceptuales, técnicos y estratégicos para la planificación y toma de desciciones en materia de impuestos diferidos. [Conceptual, technical and strategic elements for planning and making decisions regarding deferred taxes]. Recuperado de https://n9.cl/6xwc 
Revista Arbitrada Interdisciplinaria KOINONIA

Año V. Vol V. N¹0. Julio - Diciembre 2020

Hecho el depósito de Ley: FA2016000010

ISSN: 2542-3088

FUNDACIÓN KOINONIA (F.K). Santa Ana de Coro. Venezuela.

Mercedes Elizabeth Cárdenas-Pañi; Cecilia Ivonne Narváez-Zurita; Juan Carlos Erazo-Álvarez; Mireya Magdalena Torres-Palacios

Superintendencia de Compañias del Ecuador (2008). Resolución No. 08.G.DSC. [Resolution No. 08.G.DSC.]. Recuperado de https://n9.cl/br17

Tobar, G., \& Medina, N. (2018). NIC 12 impuesto a las ganancias: su relación entre un enfoque tributario frente a un enfoque contable. [IAS 12 income tax: its relationship between a tax approach versus an accounting approach]. Recuperado de https://n9.cl/ip8j8

Torres, D. (2011). Impacto tributaría de la implementación en el Ecuador de las NIIF y las NIC [Tax impact of the IFRS and IAS implementation in Ecuador.]. Recuperado de https://n9.cl/docv

Ugalde-Binda, N. (2014). Las Normas Internacionales de Información Financiera: historia, impacto y nuevos retos de la IASB. [International Financial Reporting Standards: history, impact and new challenges of the IASB]. Revista De Ciencias Económicas, 32(1), 205-216. https://doi.org/10.15517/rce.v32i1.15058

Vílchez-Olivares, P. (2007). Estado de las normas internacionales emitidas por la junta de normas internacionales de contabilidad. [Status of international standards issued by the international accounting standards board]. Quipukamayoc, 14(28), 59-66. https://doi.org/10.15381/quipu.v14i28.5364

Villegas-Escobar, D. (2018). Diseño de una guía tributaria de aplicación del impuesto diferido para la conciliación entre normas contables y normas tributarias. [Design of a tax guide to apply deferred tax for the reconciliation between accounting norms and tax norms]. Recuperado de https://n9.cl/a5ac

Zeff, S. (2012). La evolución del IASC al IASB, y los retos que enfrenta. [The evolution of the IASC to the IASB, and the challenges they face.]. The Accounting Review, 121-164. Recuperado de https://n9.cl/kf8z

(C2020 por los autores. Este artículo es de acceso abierto y distribuido según los términos y condiciones de la licencia Creative Commons Atribución-NoComercial-Compartirlgual 4.0 Internacional (CC BY-NC-SA 4.0)

(https://creativecommons.org/licenses/by-nc-sa/4.0/). 